\title{
ORT_15 - Standardization and Performance of the micro Plaque Reduction Neutralization- Horseradish Peroxidase ( $\mu$ PRN-HRP) - a Test for Quantification of Yellow Fever Antibodies
}

\author{
Marisol Simões ${ }^{1 *}$; Stephanie Almeida da Silva ${ }^{1}$; Sheila Maria Barbosa de Lima ${ }^{1}$; Luiz Antonio \\ Bastos Camacho². \\ ${ }^{1}$ Fiocruz/Bio-Manguinhos; \\ ${ }^{2}$ Fiocruz/ENSP.
}

Introduction: Yellow fever (YF) is an acute, febrile infectious disease, which remains a major public health problem, especially in endemic areas in Africa and South America. The available vaccines for YF are safe and immunogenic, inducing neutralizing antibodies that appear early, are protective and long lasting. Although it is considered the reference for the analysis of immune response to infection and to vaccination, the plaque reduction neutralization test (PRNT) is labor-intensive, time-consuming, difficult to execute, and requires skilled human resources, specific equipment and inputs. The micro-PRNT version (in 96-well plates) is more operational and has a higher sample throughput than the PRNT in 6-well plates. However, in addition to the aforementioned disadvantages, the smaller wells of micro-PRNT makes the reading of plaques more difficult. With the revelation step based on immunoenzymatic methodology and a semi-automated reading of the plates, the $\mu \mathrm{PRN}-\mathrm{HRP}$ (micro Plaque Reduction Neutralization - Horseradish Peroxidase) is a faster and more efficient test for the quantification of YF neutralizing antibodies. The performance of $\mu$ PRN-HRP shall be compared to the regular PRNT to assess its potential for routine use as a serological test in laboratory confirmation of YF and in immunogenicity studies of YF vaccines.

Objective: This work aimed to standardize, validate and finally compare the $\mu$ PRN-HRP with the reference test (PRNT, in 6-well plates) and the micro-PRNT.

Methodology: Following the definition of the execution protocol (as cell type and density, virus substrain, times of neutralization and final incubation, overlay and monoclonal antibody) attributes of precision, accuracy, selectivity and robustness were evaluated to validate the $\mu$ PRN-HRP test. Once validation was finalized, 200 sera of vaccinees were processed using $\mu$ PRN-HRP and PRNT in 96-well plates (microPRNT) to titer YF antibody levels (reciprocal dilution and mili-International Units, $\mathrm{mIU} / \mathrm{mL}$ ). The results obtained by these methods were compared with the reference test (PRNT, in 6-well plate) and their agreement was measured with the Intraclass Correlation Coefficient (ICC).

Results: The standardization of the YF $\mu$ PRN-HRP was successful, following a protocol of test execution within 4 days. The analysis criteria for all validation assays were achieved for $\mu$ PRN-HRP. Weak to moderate agreement (ICC and 95\% confidence intervals, for single measure and average measure) was observed for the $\mu$ PRN-HRP with the PRNT for titers in reciprocal dilution: $0.29(0.16-0.41) ; 0.45$ (0.27-0.58). ICC between micro-PRNT and PRNT was higher: 0.59 (0.49-0.68) and 0.74 (0.66-0.81). Using titers in $\mathrm{mIU} / \mathrm{mL}$ ICC between PRNT and $\mu$ PRN-HRP was substantial: $0.69(0.61-0.76)$ and 0.82 (0.76-0.87), whereas agreement between micro-PRNT and PRNT was inferior: 0.48 (0.37-0.58) and $0.65(0.54-0.74)$.

Conclusion: The good performance of the $\mu \mathrm{PRN}-\mathrm{HRP}$ enables it to replace the micro-PRNT as a substitute for the 6-well PRNT, in the confirmation of natural YF infection and immune response to vaccination.

Keywords: Neutralizing antibodies; Yellow fever; Neutralization test 